\title{
Molecular hydrogen gas at high-velocity associated with the Monoceros Loop SNR
}

\author{
B. Y. Welsh ${ }^{1}$, B. L. Rachford ${ }^{2}$, and J. Tumlinson ${ }^{2}$ \\ 1 Experimental Astrophysics Group, Space Sciences Laboratory, UC Berkeley, Berkeley, CA 94720, USA \\ 2 Center for Astrophysics and Space Astronomy, University of Colorado, Boulder, CO 80309, USA
}

Received 31 August 2001 / Accepted 15 October 2001

\begin{abstract}
We present Far Ultraviolet Spectroscopic Explorer (FUSE) observations of molecular hydrogen $\left(\mathrm{H}_{2}\right)$ gas observed at a high-velocity of $V_{\mathrm{LSR}}=+66 \mathrm{~km} \mathrm{~s}^{-1}$ towards the star HD 47240 which lies just behind the Monoceros Loop Supernova Remnant at a distance of $\sim 1800$ pc. These absorption features have been detected in six lines of the $J=3$ rotational level, with an equivalent molecular hydrogen column density of $\log N\left(\mathrm{H}_{2}\right)=$ $14.10, \pm 0.12 \mathrm{~cm}^{-2}$. The observed data are best fit with a single excitation temperature, $T_{\mathrm{ex}}$, of $\sim 1000 \mathrm{~K}$, which when comparised to temperatures generally found for low $J$-levels is unusually high for interstellar $\mathrm{H}_{2}$. The molecular fraction in this HV cloud has a very low value of $\log f=-4.5 \pm 0.25$, which is similar to that found for HV clouds in the galactic halo.
\end{abstract}

Key words. ISM: supernova remnants - ISM: molecules

\section{Introduction}

Although the majority of molecular hydrogen $\left(\mathrm{H}_{2}\right)$ resides in the vast galactic complexes of dense molecular clouds, much information concerning its formation and destruction mechanisms can be gained from observations of its physical state within the diffuse clouds of the general interstellar medium. The dipole-allowed electronic transitions of the Lyman and Werner bands of the $\mathrm{H}_{2}$ molecule lie in the 912-1100 A spectral region and are thus ideally suited for absorption studies using the recently launched NASA Far Ultraviolet Spectroscopic Explorer (FUSE) satellite (Moos et al. 2000). Several studies are currently being undertaken by FUSE to understand the physical state of the $\mathrm{H}_{2}$ molecule existing in a wide variety of physical environments. These include studies of $\mathrm{H}_{2}$ absorption in galactic translucent interstellar clouds (Snow et al. 2000), in the Magellanic Clouds (Tumlinson et al. 2002), in diffuse clouds of the galactic disk (Shull et al. 2000) and in clouds in the lower galactic halo (Richter et al. 2001). Even though the $\mathrm{H}_{2}$ molecule has been observed for over 30 years (Shull \& Beckwith 1982), many questions still remain concerning its detailed formation, destruction and excitation processes, especially for the higher rotation levels of $J>1$ which are generally thought to be formed by non-thermal excitation processes involving pumping by ultraviolet (UV) photons (Jura 1975).

The early-type (B1Ib) star HD $47240\left(M_{v}=6.2\right.$, $E(B-V)=0.31)$ lies just behind the Monoceros Loop

Send offprint requests to: B. Y. Welsh,

e-mail: bwelsh@ssl. berkeley.edu
Supernova Remant (SNR) at a distance of $\sim 1800 \mathrm{pc}$, and is thus well located for sampling any disturbed interstellar atomic or molecular gas associated with the SNR. Visible, near UV and far UV observations of this line-of-sight have revealed a high-velocity (HV) absorption component at $V_{\mathrm{LSR}}=+65 \mathrm{~km} \mathrm{~s}^{-1}$ associated with an expanding gas cloud of the SNR (Sfeir 1999; Welsh et al. 2001, hereafter Paper I). For the purpose of this Paper we define an absorption component with a velocity $>50 \mathrm{~km} \mathrm{~s}^{-1}$ as being "high-velocity", in contrast with the more widely accepted definition used for HV clouds in the halo. This HV feature was detected towards HD 47240 in several UV atomic lines with ionization potentials ranging up to $23.3 \mathrm{eV}$, and high spectral resolution visible observations reveal a more complex absorption structure (with at least two velocity components at $V_{\mathrm{LSR}}=+60$ and $\left.+71 \mathrm{~km} \mathrm{~s}^{-1}\right)$, suggesting that it is composed of several ionized and neutral gas shells expanding at slightly different velocities.

In this Paper we report on FUSE observations of $\mathrm{HV}$ molecular $\mathrm{H}_{2}$ line absorption seen at $V_{\mathrm{LSR}}=+66 \mathrm{~km} \mathrm{~s}^{-1}$ towards HD 47240. High-velocity molecular $\mathrm{H}_{2}$ absorption has been previously observed by FUSE towards stars in the outer galactic halo which are associated with the Magellanic Stream (Sembach et al. 2002) and towards an intermediate-velocity halo cloud (Richter et al. 2001). In addition, Gringel et al. (2000) have reported ORFEUS - SPAS - II detections of $\mathrm{HV}$ molecular $\mathrm{H}_{2}$ at a velocity of $-62 \mathrm{~km} \mathrm{~s}^{-1}$ towards the halo star HD 93521. However, detections of $\mathrm{H}_{2}$ at similarly high velocities in the galactic disk are very rare and have thus far only 
been reported towards the star $\zeta$ Orionis by Jenkins and Peimbert (1997). It was found that as the $J$-level increased from 0 to 5 the width of the velocity profile of the individual molecular absorption lines also increased, together with a small drift of the line profiles' center towards more negative velocities. In order to explain this anomalous behavior, Jenkins and Peimbert proposed that the high velocity $\mathrm{H}_{2}$ molecules were most probably formed in postshock gas undergoing further compression as it recombined and cooled. However, in the Copernicus satellite survey of galactic $\mathrm{H}_{2}$, Spitzer \& Morton (1976) proposed that the most negative velocity components probably arose in thin, dense sheets in cold compressed regions that followed interstellar shock fronts arising from SN blast waves and/or the expansion of stellar HII regions. We recall that molecular hydrogen is thought to form on interstellar dust grains and is then subsequently ejected from the grain surface into the ISM. Thus, since SNRs (such as the Monoceros Loop) are normally associated with shockwaves that interact with ambient (cold) interstellar material, the HD 47240 line-of-sight is an ideal test-site for probing the formation of cold molecular gas that may be influenced by HV shocks. This is particularly important since it has long been suspected that SNRs can trigger the formation of new molecular clouds and thus initiate the process of star formation.

\section{Observations and data processing}

Details of the FUSE observations and data processing techniques used on the spectral data gained in the LiF channels (990-1187 $\AA$ ) towards HD 47240 have been detailed in Paper I. Further FUSE observations of this object were obtained over the course of 3 orbits on March 4th 2000, in which data in the SiC spectral channels (912-1000 А) were obtained with the star centered in the $30 \times 30$ arcsec focal plane aperture. Both spectral data sets have been individually processed using version 1.8.7 of the FUSE science data reduction (CALFUSE) pipeline, details of which can be found in Sahnow et al. (2000).

The processed far UV spectrum of HD 47240 (912$1187 \AA$ ) was then used to search for the presence of individual $\mathrm{H}_{2}$ molecular lines using the wavelengths listed for the Lyman and Werner bands given in Abgrall et al. (1993a, 1993b). The resultant spectral resolution of the data (see Paper I) was $13 \mathrm{~km} \mathrm{~s}^{-1}$ and the typical $\mathrm{S} / \mathrm{N}$ of the spectra were $\sim 20: 1$. All detected $\mathrm{H}_{2}$ line profiles were then inspected for the presence of associated HV components. Unfortunately it was not possible to detect $\mathrm{HV}$ components in the $J=0$ and $J=1$ bands due to the central line profile being strongly damped and thus obscuring any $\mathrm{HV}$ features with a velocity $<80 \mathrm{~km} \mathrm{~s}^{-1}$ with respect to the main component.

Inspection of the $J=2-5$ lines revealed that a $\mathrm{HV}$ component was clearly visible in some of these profiles. In order to proceed further with the detailed extraction of these profiles an accurate wavelength scale needed to be applied since the FUSE spectral data has an inherent

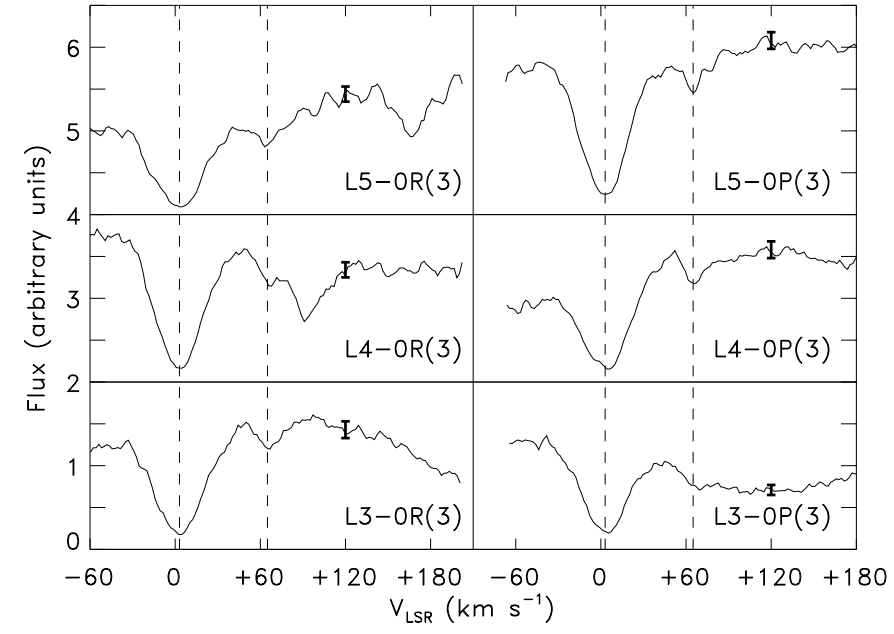

Fig. 1. Six of the $J=3$ molecular $\mathrm{H}_{2}$ absorption lines that exhibit a HV component at $V_{\mathrm{LSR}}=+66 \mathrm{~km} \mathrm{~s}^{-1}$ (marked with dashed line). The other dashed line marks the strong central absorption at $V_{\mathrm{LSR}}=+3 \mathrm{~km} \mathrm{~s}^{-1}$. The small solid bar indicates a typical 2-sigma error to the continuum level noise.

non-linear wavelength error typically of $10-20 \mathrm{~km} \mathrm{~s}^{-1}$. We have assumed that all the main components of the $\mathrm{H}_{2}$ lines occur at $V_{\mathrm{LSR}}=+3 \mathrm{kms}^{-1}$, which is the same velocity as that of the visible $\mathrm{CH}$ molecular line at $4232.5 \AA$ as observed by Sfeir (1999).

\subsection{Interstellar analysis}

In Fig. 1 we show six lines of the $J=3$ level that clearly show an unambiguous detection of a $\mathrm{HV}$ component at $V_{\mathrm{LSR}}=+66 \mathrm{~km} \mathrm{~s}^{-1}$. For each of these six lines we have fitted the local stellar continuum with a multi-order polynomial to produce a residual intensity profile. No individual $J=2$ or $J=4$ lines showed HV component detections at a level $>1 \sigma$. However, to improve the resultant $\mathrm{S} / \mathrm{N}$ of each of the $J=2$ and $J=4$ levels (and even the welldetected $J=3$ level) we have co-added five $J=2$, six $J=3$ and seven $J=4$ individual line profiles to produce the resultant profile plots for an "average" $J=2,3$ and 4 molecular $\mathrm{H}_{2}$ line as shown in Fig. 2. As a guide to the eye we have overplotted the expected position of both the $V=+3 \mathrm{~km} \mathrm{~s}^{-1}$ main component and the $V=+66 \mathrm{~km} \mathrm{~s}^{-1}$ HV component with dashed lines. The average separation between the two well detected components for the six, individual $J=3$ lines is $\delta V=+63.3 \pm 1.4 \mathrm{~km} \mathrm{~s}^{-1}$. Note that we also show the magnitude of a $2 \sigma$ error in the flux (dark error bars) and that the co-added $J=4$ spectrum now reveals a marginal detection of the $\mathrm{HV}$ component that is comparable in strength to the error bar.

Using these data we have proceeded with a detailed analysis of the six $J=3$ lines listed in Table 1 . In this table, for completeness, we also list the $J=2$ and $J=4$ lines used to produce the co-added spectra discussed previously. We have used the curve-of-growth analysis described in detail by Tumlinson et al. (2002) to derive a column density, $N(J)$, and an associated Doppler $b$ value for each 
Table 1. High-velocity molecular $\mathrm{H}_{2}$ lines observed towards HD 47240.

\begin{tabular}{|c|c|c|}
\hline Line Identification & EW of HV component $(m \AA)$ & $\log N\left(\mathrm{~cm}^{-2}\right)$ \\
\hline L5-OR(3) $\lambda 1041.16 \AA$ & $17.4 \pm 5.0$ & $14.06 \pm 0.15$ \\
\hline L5-OP(3) $\lambda 1043.50 \AA$ & $16.6 \pm 3.3$ & $14.21 \pm 0.10$ \\
\hline L4-OR(3) $\lambda 1053.98 \AA$ & $10.1 \pm 2.6$ & $13.89 \pm 0.13$ \\
\hline L4-OP(3) $\lambda 1056.47 \AA$ & $11.0 \pm 3.9$ & $14.07 \pm 0.19$ \\
\hline L3-OR(3) $\lambda 1067.48 \AA$ & $13.4 \pm 3.3$ & $14.12 \pm 0.12$ \\
\hline $\mathrm{L} 3-\mathrm{OP}(3) \lambda 1070.14 \AA$ & $12.9 \pm 2.2$ & $14.23 \pm 0.08$ \\
\hline W0-OQ(2) $\lambda 1010.94 \AA$ & $<10$ & $<13.56$ \\
\hline $\mathrm{L} 7-\mathrm{OP}(2) \lambda 1016.46 \AA$ & $<10$ & $<13.56$ \\
\hline $\mathrm{L} 5-\mathrm{OP}(2) \lambda 1040.37 \AA$ & $<10$ & $<13.56$ \\
\hline $\mathrm{L} 4-\mathrm{OP}(2) \lambda 1053.28 \AA$ & $<10$ & $<13.56$ \\
\hline L3-OP(2) $\lambda 1066.90 \AA$ & $<10$ & $<13.56$ \\
\hline L7-OP(4) $\lambda 1023.43 \AA$ & $<10$ & $<13.34$ \\
\hline $\mathrm{L} 6-\mathrm{OR}(4) \lambda 1032.35 \AA$ & $<10$ & $<13.34$ \\
\hline $\mathrm{L} 6-\mathrm{OP}(4) \lambda 1035.18 \AA$ & $<10$ & $<13.34$ \\
\hline $\mathrm{L} 5-\mathrm{OR}(4) \lambda 1044.54 \AA$ & $<10$ & $<13.34$ \\
\hline L4-OR(4) $\lambda 1057.38 \AA$ & $<10$ & $<13.34$ \\
\hline L4-OP(4) $\lambda 1060.58 \AA$ & $<10$ & $<13.34$ \\
\hline L3-OP(4) $\lambda 1074.31 \AA$ & $<10$ & $<13.34$ \\
\hline
\end{tabular}

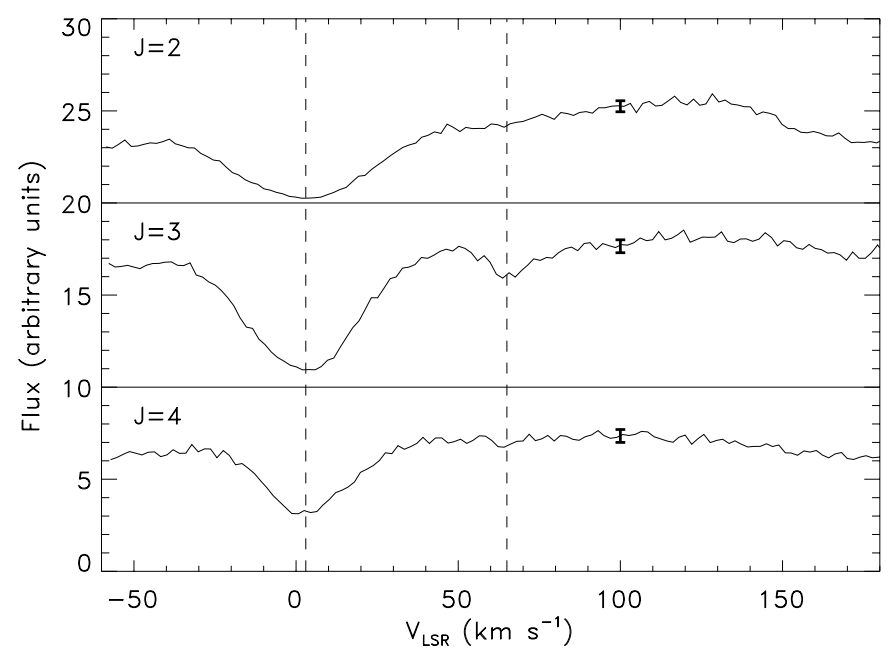

Fig. 2. Profiles of the co-added $J=2,3$ and 4 individual molecular $\mathrm{H}_{2}$ lines. The $\mathrm{HV}$ component is clearly seen at $V_{\mathrm{LSR}}=+66 \mathrm{kms}^{-1}$ for the case of the (six) co-added $J=3$ lines and is marginally detected at the one- $\sigma$ level for the seven co-added $J=4$ lines. The solid bar corresponds to a $2-\sigma$ error.

of the $J=3 \mathrm{HV}$ line components. We have assumed a single-component Maxwellian curve of growth and minimized the residuals about the best-fit curve for the lines within each level. All lines (and HV component upper limits) were assumed to be on the linear part of the curve of growth (i.e. $b>5 \mathrm{kms}^{-1}$, as suggested by the data). From the values listed in Table 1 we have derived an average column density, $\log N(3)=14.10 \pm 0.12 \mathrm{~cm}^{-2}$ for all of the $J=3$ lines. In addition, we derive upper limits of $\log N(2)<13.56 \mathrm{~cm}^{-2}$ and $\log N(4)<13.34 \mathrm{~cm}^{-2}$ for the $\mathrm{HV}$ components associated with co-added spectra of the $J=2$ and $J=4$ levels respectively.

\section{Results and discussion}

In Fig. 3 we show an excitation diagram for the HV component based on our measured $J=3$ level column density and upper limits for the $J=2$ and 4 levels. This figure shows the column density of each level divided by its statistical weight plotted against excitation energy $(\mathrm{eV})$. The slope of a line in this diagram corresponds to a particular excitation temperature via the relationship, $T_{\text {ex }}=5040 /$ slope (Spitzer et al. 1974). As we only have a detection for a single rotational state we therefore cannot derive a formal value of $T_{\text {ex }}$. However, the upper limits for the $J=2$ and $4 \mathrm{HV}$ components provide interesting constraints on this quantity. Figure 3 shows two possibilities for the slope of the best-fit line, corresponding to the 2- $\sigma$ and 4- $\sigma$ upper limits for $N(J=2)$ which result in excitation temperatures of $510 \mathrm{~K}$ and $1650 \mathrm{~K}$ respectively. The 2- $\sigma$ upper limit gives a fit that is more consistent with the possible detection of a $J=4 \mathrm{HV}$ component in the co-added spectrum. Thus, we favor an excitation temperature of $\sim 1000 \mathrm{~K}$ for the HV component. This high value of molecular excitation temperature is comparable to the highest temperature found in the Copernicus survey of the galactic disk, but is an order of magnitude higher than the average value of $77 \pm 17 \mathrm{~K}$ found for $J=0,1$ lines in the disk survey (Savage et al. 1977). The (two) lines-ofsight with similarly high $T_{\text {ex }}$ values found by Copernicus were associated with relatively small $\mathrm{H}_{2}$ column densities, although most lines-of-sight with such low column densities did not show such unusually high excitation temperatures. We also note that elevated excitation temperatures for high- $J$ levels (as opposed to much lower temperatures of $\sim 100 \mathrm{~K}$ found for low $J$-levels) have recently been found by the FUSE satellite towards two galactic translucent 
clouds and in the Large Magellanic Cloud (Snow et al. 2000; Rachford et al. 2001; Friedman et al. 2000). A variety of physical processes that include fluorescent pumping by incident (low level) UV radiation, interstellar shocks and the formation of $\mathrm{H}_{2}$ on dust grains have all been forwarded to explain this elevated temperature effect for high rotational levels of molecular $\mathrm{H}_{2}$ (Shull \& Beckwith 1982).

Although we have not been able to determine column densities for the $\mathrm{HV}$ components of the $J=0$ or 1 states, we can make a rough estimate of the total $\mathrm{H}_{2}$ column density from our excitation analysis. We note that a single excitation temperature for all $J$-levels was found for all linesof-sight with small $N(\mathrm{H})_{2}$ values in the Copernicus survey (Spitzer et al. 1974), and for simplicity in our present analysis we assume that rotational states that contribute significantly to the total molecular hydrogen column density can also be described by a single excitation temperature. We note, however, that recent FUSE results require multiple temperature fits to the molecular $\mathrm{H}_{2}$ line data and the line-of-sight to this SNR is probably not typical of those found in the general ISM. Thus bearing these caveats in mind, if we sum over all the $J=0,7$ lines we obtain a value of $\log N\left(\mathrm{H}_{2}\right) \sim 14.7 \mathrm{~cm}^{-2}$. This value (which is dominated by the contribution from the $J=3$ state) is a firm lower limit to $N\left(\mathrm{H}_{2}\right)$ and can most probably be associated with an error estimate of $\sim 30 \%$ (i.e. $\left.\log N\left(\mathrm{H}_{2}\right)_{\text {tot }} \sim 14.7 \pm 0.13 \mathrm{~cm}^{-2}\right)$. We also note that the far larger column densities for the $J=0$ and 1 states for the low temperature case are offset by the larger contribution from $J>3$ in the high temperature case. When combined with a value of $\log N(\mathrm{HI})=19.2 \pm 0.2 \mathrm{~cm}^{-2}$ for the $\mathrm{HV}$ atomic component (derived from the HV component seen in the SII lines, Paper I), we derive a corresponding molecular fraction of $\log f=-4.5 \pm 0.25$. This value is relatively low for a line-of-sight through the galactic disk, but is very similar to that found for HV gas associated with clouds in the halo (Richter et al. 2001; Gringel et al. 2000). Both of these sight-lines into the halo possess low levels of interstellar dust and have an elemental abundance of $\log [\mathrm{Fe} / \mathrm{HI}] \sim-0.65$ for a $\mathrm{HV}$ component with a velocity $\sim-60 \mathrm{~km} \mathrm{~s}^{-1}$. We note that almost identical conditions $\left(\log [\mathrm{Fe} / \mathrm{HI}]=-0.96\right.$ and $\left.V=+65 \mathrm{~km} \mathrm{~s}^{-1}\right)$ are to be found for the Monoceros Loop HV cloud! For the halo HV clouds the influence of nonthermal excitation by fluorescent UV photon pumping has been forwarded to explain the formation of the high- $J$ states of $\mathrm{H}_{2}$. However, the presence of a SNR shock seems a more likely explanation for the $\mathrm{HV}$ components of the $J>1$ rotational states seen towards the Monoceros Loop. This view is supported in part by the recent discovery of a pervasive warm molecular gas component of the diffuse ISM in which the $\mathrm{H}_{2}$ excitation of the $J>2$ levels is best explained by the presence of collisional excitation (Gry et al. 2002). To date, the interaction between SNRs and ambient molecular clouds has been reported for only a few sources using observations of CO emission lines (Koo et al. 2001), and thus our present detection of $\mathrm{HV}$ molecular $\mathrm{H}_{2}$ lines in

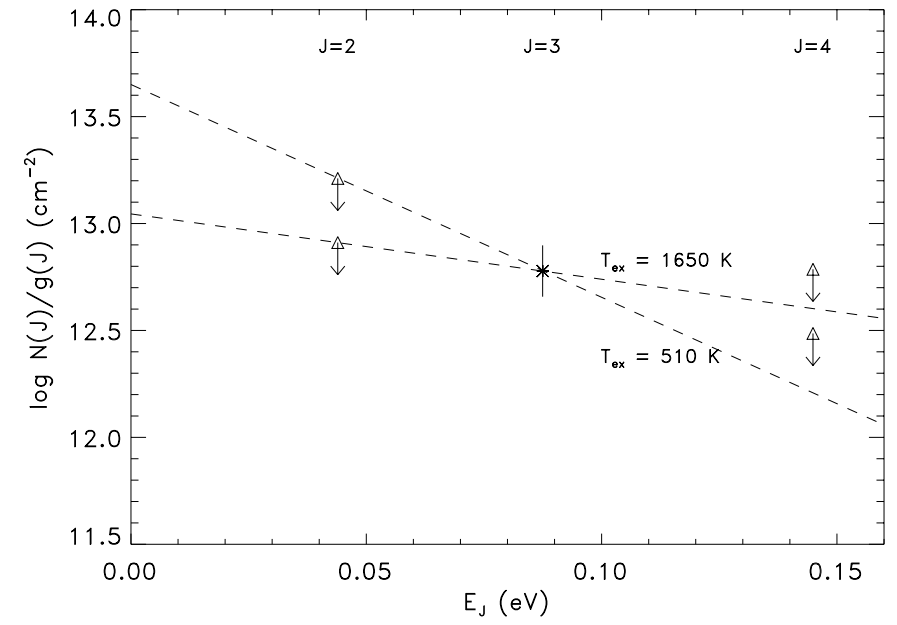

Fig. 3. Excitation diagram for the $\mathrm{HV}$ component of the $\mathrm{H}_{2}$ lines. The derived column density for the $J=3$ level is shown, along with $2-\sigma$ and $4-\sigma$ upper limits on the $J=2$ and 4 levels. The dashed lines give two possible excitation temperatures, as described in the text.

absorption may prove important for testing the predictions of shock/cloud collision models (Klein et al. 1994).

\section{Conclusion}

Observations with the NASA FUSE satellite have revealed high-velocity components at $V_{\mathrm{LSR}}=+66 \mathrm{~km} \mathrm{~s}^{-1}$ in six of the $J=3$ rotational states of interstellar molecular hydrogen observed towards the star HD 47240 which lies just behind the Monoceros Loop SNR. We have derived a best-fit excitation temperature, $T_{\mathrm{ex}}$, of $\sim 1000 \mathrm{~K}$ to the data, based on the $J=3$ level detections and upper limits to the HV components of the $J=2$ and 4 states. The molecular fraction in this $\mathrm{HV}$ cloud has a very low value of $\log f=-4.5 \pm 0.25$, similar to values found for HV clouds in the galactic halo. Production mechanisms such as UV fluorescent pumping and interstellar shocks have been forwarded to explain the formation of the high- $J$ rotational states of the $\mathrm{H}_{2}$ molecule. It would be of great interest to observe other SNRs (such as Puppis-A and Shajn 147) to see whether HV molecular gas is similarly detected at high velocities in the high rotational levels, such that the presence of SNR shock collisions can be shown to be an important influence on the formation of $\mathrm{H}_{2}$ throughout the galaxy.

Acknowledgements. This work is based on data obtained for the Guaranteed Time science team by the NASA-CNES-CSA FUSE Mission operated by the Johns Hopkins University. Financial support to U.S. particpants has been provided by the NASA contract NAS5-32985. Particular thanks are extended to Prof. Ted Snow for very useful discussions and important suggestions that have greatly improved this paper.

\section{References}

Abgrall, H., Roueff, E., Launay, F., Roncin, J. Y., \& Subtil, J. L. 1993a, A\&AS, 101, 273 
Abgrall, H., Roueff, E., Launay, F., Roncin, J. Y., \& Subtil, Richter, P., Savage, B. D., Wakker, B. P., Sembach, K. R., \& J. L. 1993b, A\&AS, 101, 323

Kalberla, P. M. W., 2001, ApJ, 549, 281

Friedman, S., Howf, J. C., Anderson, B.-G., et al. 2000, ApJ, Sahnow, D., Moos, H. W., Ake, T. B., et al., 2000, ApJ, 538, L7 538, L39

Gringel, W., Barnstedt, J., de Beer, K. S., et al., 2000, A\&A, 358, L37

Savage, B., Bohlin, R., Drake, J., \& Budich, W. 1977, ApJ, 216, 291

Sembach, K., et al. 2002, ApJ

Gry, C., Boulanger, C., Nehme, C., \& Pineau des Forets, G. 2002, A\&A, in press

Jenkins, E. B., \& Peimbert, A. 1997, ApJ, 477, 265

Jura, M. 1975, ApJ, 197, 575

Klein, R., McKee, C., \& Colella, P. 1994, ApJ, 420, 213

Koo, B.-C., Rho, J., Reach, W. T., Jung, J., \& Mangum, J. G. 2001, ApJ, 552, 175

Sfeir, D. M. 1999, Ph.D. Thesis, University of Paris 6

Shull, J. M., \& Beckwith, S. V. 1982, ARA\&A, 20, 163

Shull, J. M., Tumlinson, J., Jenkins, E. B., et al. 2000, ApJ, 538, L73

Snow, T. P., Rachford, B. L., Tumlinson, J., et al. 2000, ApJ, 538, L65

Moos, H. W., Cash, W. C., Cowie, L. L., et al. 2000, ApJ, 538, L1

Spitzer, L., Cochran, W., \& Hirshfeld, A. 1974, ApJS, 28, 373

Spitzer, L., \& Morton, W. A. 1976, ApJ, 204, 731

Tumlinson, J., et al. 2002, ApJ, in press

Rachford, B. L., Show, T. P., Tumlinson, J., et al. 2001, ApJ, 555,839

Welsh, B. Y., Sfeir, D., Sallmen, S., \& Lallement, R. 2001, A\&A, 372, 516 